\title{
Etiology and Types of Seizures in Patients Presenting to a Tertiary Care Hospital in Karachi: A Cross-Sectional Study
}

\author{
Wajid Jawaid $^{1}$, Qamar Nisa ${ }^{1}$, Sumera R. Umer ${ }^{1}$, Sidra J. Barry ${ }^{1}$, Amir Qureshi ${ }^{1}$, Naila N. Shahbaz ${ }^{1}$ \\ 1. Neurology, Dow University of Health Sciences, Karachi, PAK
}

Corresponding author:Wajid Jawaid, wjawaid85@gmail.com

\section{Abstract}

\section{Introduction}

Epilepsy is a burdensome disorder for affected individuals and community. There is limited data available on the epidemiological aspects of seizures in Pakistan and further research is necessary. We aimed to fill this gap by studying this information in epilepsy patients presenting to our neurology department. The purpose of this study is to evaluate the causes and types of seizures among the target population.

\section{Method}

This is a cross-sectional study conducted at the Department of Neurology, Dr. Ruth K.M. Pfau Civil Hospital Karachi. In this study we evaluated the causes and types of seizures among patients presenting to our department during the two-year study duration (January 2018-December 2019). Informed consent was taken. Detailed history was taken including features of seizure episodes, age at first seizure, family history and comorbid conditions. Relevant investigations were carried out. The data was compiled to deduce the relevant information using SPSS v20.0. T-test and Chi-square were used for analyzing the data.

\section{Results}

A total of 996 patients presented during the study duration. Primary seizures were found in $58 \%$ cases while secondary seizures were found in $42 \%$ cases. This distribution was more equal in children with $49.6 \%$ primary seizures and $50.4 \%$ secondary seizures; the gap widened in adults with $64.3 \%$ primary seizures and $35.7 \%$ secondary seizures. The most common cause of secondary seizures was neonatal encephalopathy which was present in $18.7 \%$ patients, followed by traumatic head injury in $18.2 \%$ patients. Central nervous system (CNS) infection was the cause in $17.9 \%$ patients, cerebral tumors in $14.1 \%$ patients, stroke in $11.5 \%$, metabolic encephalopathy in $7.4 \%$, febrile seizures in $6.5 \%$ and CNS malformations in $5.7 \%$ patients. The top three causes in children were neonatal encephalopathy (28.3\%), CNS infections (19.3\%) and febrile seizures (12.7\%). Adults with secondary seizures were diagnosed most often with head trauma (25.2\%), cerebral tumors (19.9\%) and stroke (18.4\%) as causative factors. The most common type of seizures was generalized onset tonic-clonic seizures which was found in $73.0 \%$ patients followed by focal to bilateral tonic-clonic seizures in $8.9 \%$ patients. Other types of seizures included focal aware seizures in $5.0 \%$, mixed seizure types in $4.2 \%$, focal impaired awareness seizures in $3.1 \%$, absence seizures in $2.7 \%$, myoclonic seizures in $2.0 \%$ and atonic seizures in $1.0 \%$ patients. Seizures in children were mostly generalized onset tonic-clonic seizures (75.4\%), mixed seizure types (5.7\%) and focal to bilateral tonic-clonic seizures (5.2\%). In adults the three most common types corresponded to the overall result: generalized onset tonic-clonic seizures (71.2\%), focal to bilateral tonic-clonic seizures (11.6\%) and focal aware seizures (6.6\%).

\section{Conclusion}

We found that the most common cause of seizures overall in our study population was primary seizures, though primary and secondary seizures were more evenly present in children. Among secondary causes neonatal encephalopathy stood out as the most common cause in children; head trauma was the predominant cause in adults. Most common type of seizures overall and in adults was generalized onset tonic-clonic seizures, followed by focal to bilateral tonic-clonic and focal aware seizure types. Pediatric patients presented most often with generalized onset tonic-clonic seizures, followed by mixed seizure types and focal to bilateral tonic-clonic seizures.

Categories: Neurology

Keywords: phenomenology of epilepsy, primary epilepsy, secondary epilepsy, epilepsy in pakistan, epilepsy

\section{Introduction}

Approximately $10 \%$ of the world's population experience seizures at least once in life [1]. Seizures need appropriate investigations to find the underlying cause. Individuals who have two or more unprovoked seizures are diagnosed with epilepsy which is one of the most common neurological disorders. It affects 


\section{Cureus}

around 70 million people worldwide [2]. Pakistan had 1.38 million cases according to Aziz et al. in 1994. They found the prevalence of epilepsy in Pakistan as 9.99 per 1000 people [3]. There is scarce data available on the epidemiological aspects of seizures and other neurological conditions in Pakistan and further research is badly needed. We aimed to fill this gap by studying this information in all epilepsy patients presenting to neurology department, over a period of two years, in Dr. Ruth K.M. Pfau Civil Hospital Karachi.

The causes of seizures are diverse. They can be primary, or secondary with vascular, infective, metabolic, neoplastic, degenerative and genetic causes. Head injuries can also cause seizures either immediately or many years later. In the elderly, stroke and neurodegenerative conditions frequently lead to seizures [4].

The aim of this study is to evaluate the causes and types of seizures among patients presenting to a tertiary care hospital in Karachi. Current data in this regard will help the policy makers in ensuring availability of the best drugs based on seizure types and causes. By identifying the most common causes of epilepsy, this study also intends to create opportunity and awareness for preventing these causes.

\section{Materials And Methods}

This is a cross-sectional study conducted at the Department of Neurology, Dr. Ruth K.M. Pfau Civil Hospital Karachi. In this study we evaluated the causes of seizures among patients presenting to our department during the two-year study duration (January 2018 to December 2019). The study was approved by the institutional review board. Trained doctors were asked to interview the patients after taking informed consent. Demographic variables such as age, gender and socio-economic status were recorded. Detailed history was taken including features of seizure episodes, age at first seizure, family history and co-morbid conditions. Complete physical examination was performed. Baseline investigations were ordered such as complete blood picture, liver function tests, renal function tests, serum electrolytes, serum urea and creatinine levels. Causes of seizures were evaluated using detailed history and diagnostic modalities such as electroencephalogram (EEG), magnetic resonance imaging (MRI) brain, computed tomography (CT) brain scan, and cerebrospinal fluid (CSF) sampling. MRI was performed in majority of patients while CT scan was done in only a few patients in whom MRI was not necessary for diagnosis. CSF studies were reserved for those patients in whom infective or inflammatory causes were suspected. Seizures were diagnosed and classified based on the criteria defined by International League Against Epilepsy commission on classification and terminology of seizures [5]. Patients of any age with generalized onset tonic-clonic, focal aware, focal impaired awareness, focal to bilateral tonic-clonic, myoclonic, atonic or absence seizures were included in the study. Patients with conversion disorder or psychogenic non-epileptic seizures were not included in the study. Data was analyzed using SPSS v20.0 (IBM Corp., Armonk, NY). T-test and Chi-square were used for analysis. P-values less than 0.05 were considered significant.

\section{Results}

A total of 996 patients presented during the study duration. This makes $10.5 \%$ of the total number of 9444 patients who presented to neurology department in this period. The mean age of our patients was $21.4 \pm 3.2$ years. Among them 534 (53.6\%) were males and 462 (46.4\%) were females (Figure 1).

\section{Gender distribution}

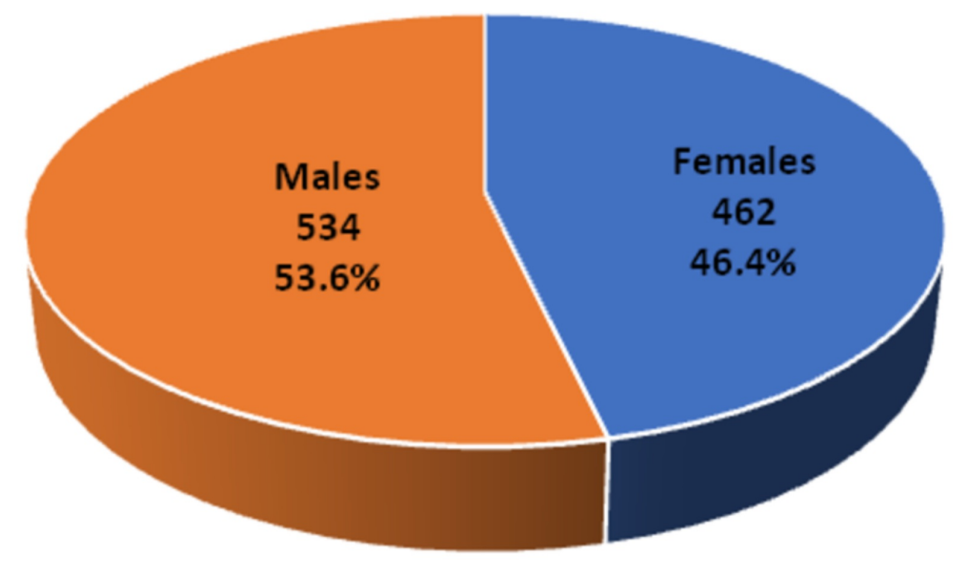




\section{Cureus}

FIGURE 1: Gender Distribution

Primary seizures were found in 578 (58\%) of total cases while secondary seizures were found in 418 (42\%) cases. Among pediatric patients primary and secondary seizures were more evenly distributed - primary seizures 207 (49.6\%) and secondary seizures 212 (50.4\%). Adult patients with primary seizures were 371 (64.3\%) and 206 (35.7\%) adults presented with secondary seizures (Figures 2-4).

\section{Causes of seizures}

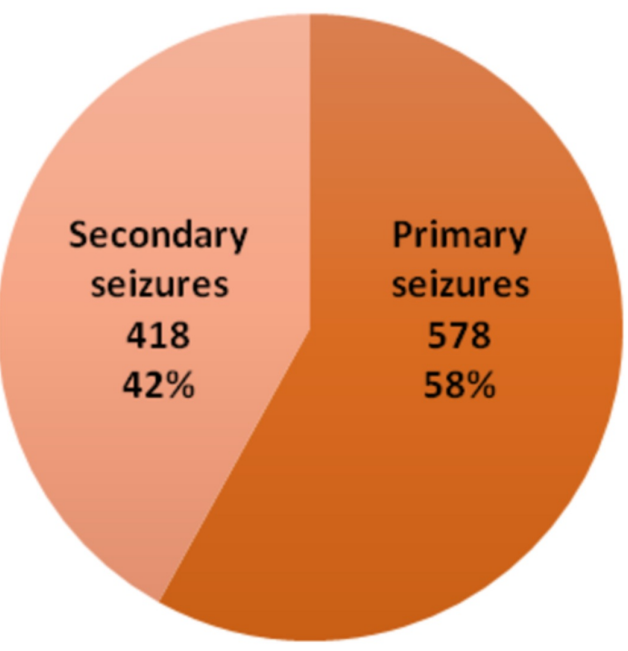

FIGURE 2: Causes of Seizures in All Patients

\section{Pediatric age group causes}

FIGURE 3: Causes of Seizures in Pediatric Patients 


\section{Cureus}

\section{Adult age group causes}

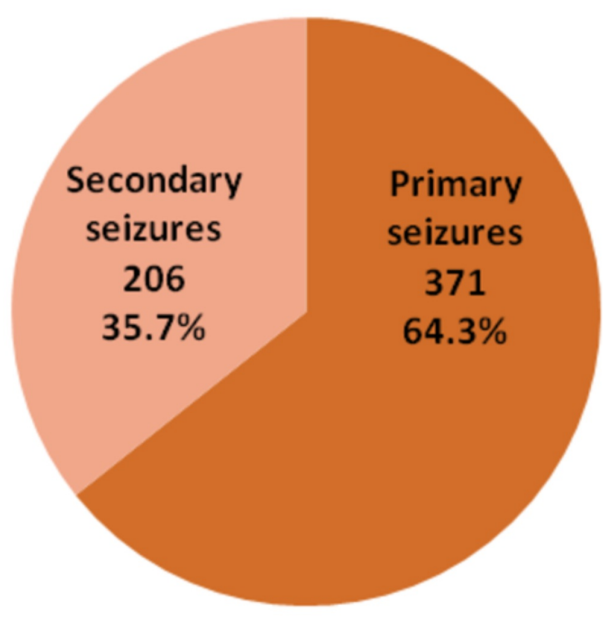

FIGURE 4: Causes of Seizures in Adult Patients

The age distribution is shown in Table 1. 


\section{Cureus}

\begin{tabular}{|c|c|}
\hline AGE GROUP & NUMBER OF PATIENTS \\
\hline Pediatric (less than 18 years) & 419 \\
\hline Adult (18 years or more) & 577 \\
\hline AGE RANGE & NUMBER OF PATIENTS \\
\hline 0 to 5 years & 115 \\
\hline 6 to 10 years & 67 \\
\hline 11 to 15 years & 87 \\
\hline 16 to 20 years & 191 \\
\hline 21 to 25 years & 121 \\
\hline 26 to 30 years & 97 \\
\hline 31 to 35 years & 41 \\
\hline 36 to 40 years & 22 \\
\hline 41 to 45 years & 42 \\
\hline 46 to 50 years & 45 \\
\hline 51 to 55 years & 62 \\
\hline 56 to 60 years & 52 \\
\hline More than 60 years & 54 \\
\hline TOTAL NUMBER OF PATIENTS & 996 \\
\hline
\end{tabular}

\section{TABLE 1: Age Distribution}

Overall the most common cause of secondary seizures was neonatal encephalopathy which was the cause in $18.7 \%(n=78)$ patients, followed by traumatic head injury in $18.2 \%(n=76)$ patients. CNS infection was the cause in $17.9 \%(n=75)$ patients, cerebral tumors in $14.1 \%(n=59)$ patients, stroke in $11.5 \%(n=48)$,

metabolic encephalopathy in $7.4 \%(n=31)$, febrile seizures in $6.5 \%(n=27)$ and cerebral malformations in $5.6 \%(n=24)$ patients. The foremost cause of secondary seizures in pediatric population was neonatal encephalopathy which was found in $28.3 \%(n=60)$, followed by CNS infections in $19.3 \%(n=41)$. Febrile seizure was the cause in $12.7 \%(n=27)$, head trauma in $11.3 \%(n=24)$, cerebral tumors in $8.5 \%(n=18)$, cerebral malformations in $8.5 \%(n=18)$, metabolic encephalopathy in $6.6 \%(n=14)$ and stroke in $4.7 \%(n=$ 10). Most prominent among the causes of secondary seizures in adults was head trauma which was found in $25.2 \%(n=52)$, followed by cerebral tumors in $19.9 \%(n=41)$ patients. Other causes were stroke in $18.4 \%$ ( $n$ $=38)$, CNS infections in $16.5 \%(n=34)$, neonatal encephalopathy in $8.7 \%(n=18)$, metabolic encephalopathy in $8.2 \%(n=17)$ and cerebral malformations in $2.9 \%(n=6)$ patients (Figures $5-7)$.

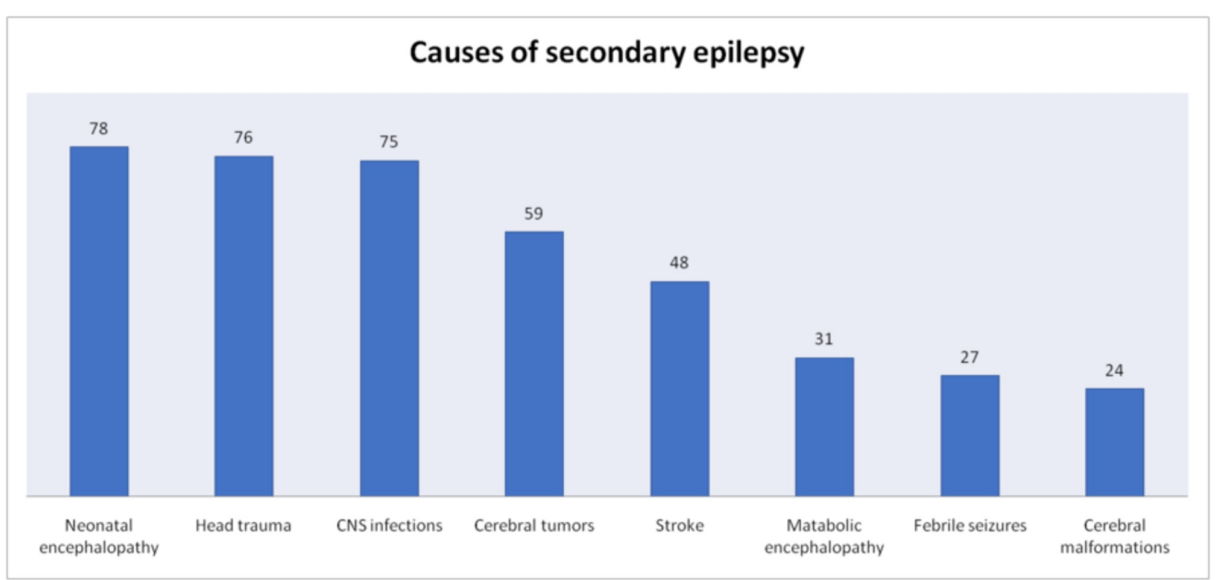




\section{Cureus}

FIGURE 5: Causes of Secondary Seizures in All Patients

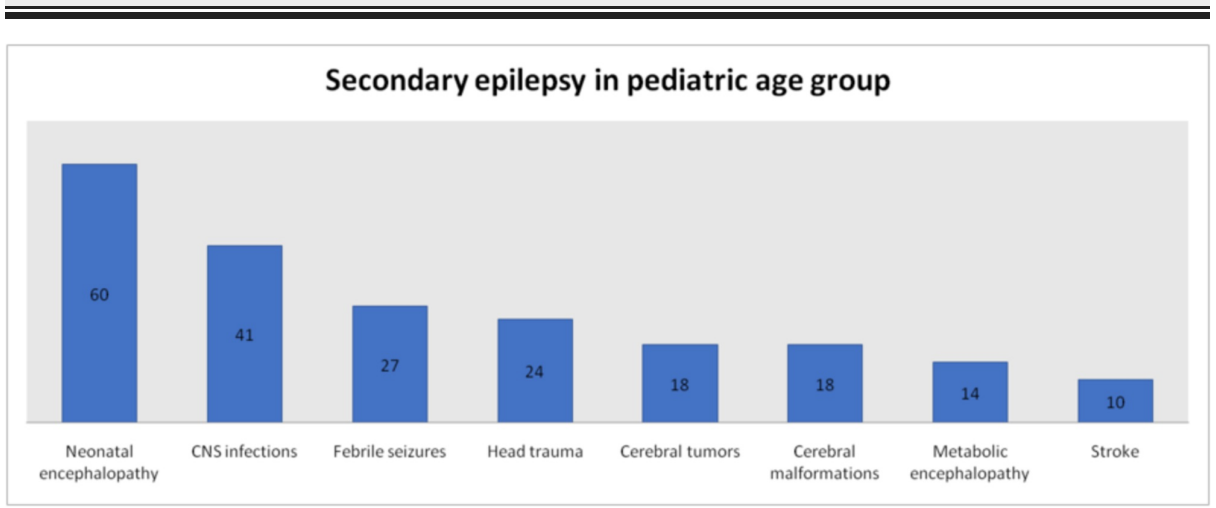

FIGURE 6: Causes of Secondary Seizures in Pediatric Patients

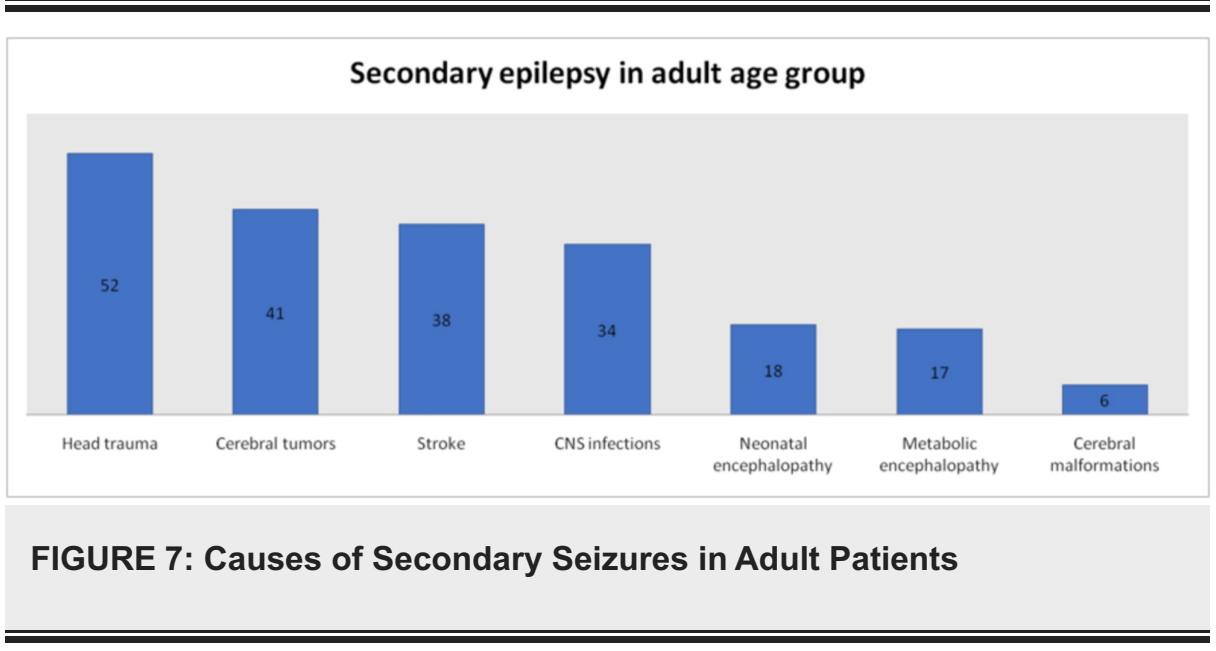

The most common type of seizures overall was generalized onset tonic-clonic seizures which was found in $73.0 \%(n=727)$ patients, followed by focal to bilateral tonic-clonic seizures in $8.9 \%(n=89)$ patients. Other types of seizures found in our study population included focal aware seizures in $5 \%(n=50)$, mixed seizure types in $4.2 \%(n=42)$, focal impaired awareness seizures in $3 \%(n=31)$, absence seizures in $2.7 \%(n=27)$, myoclonic seizures in $2 \%(n=20)$ and atonic seizures in $1 \%(n=10)$ patients. Among the pediatric population most common type of seizures was generalized onset tonic-clonic seizures in $75.4 \%(n=316)$, followed by mixed seizure types in $5.7 \%(n=24)$. Other seizure types in pediatric patients were focal to bilateral tonic-clonic in 5.2\% ( $(n=22)$, absence seizures in $4.8 \%(n=20)$, focal aware seizures in $2.9 \%(n=12)$, myoclonic seizures in $2.9 \%(n=12)$, atonic seizures in $1.9 \%(n=8)$ and focal aware seizures in $1.2 \%(n=5)$. Among the adult patients most common type of seizures was generalized onset tonic-clonic seizures in $71.2 \%(n=411)$, followed by focal to bilateral tonic-clonic seizures $11.6 \%(n=67)$. Other seizure types in adults were focal aware seizures in $6.6 \%(n=38)$, focal impaired awareness seizures in $4.5 \%(n=26)$, mixed seizure types in $3.1 \%(n=18)$, myoclonic seizures in $1.4 \%(n=8)$, absence seizures in $1.2 \%(n=7)$ and atonic seizures in $0.3 \%(n=2)$ (Tables 2-4). 


\section{Cureus}

TOTAL NUMBER OF PATIENTS

Generalized onset tonic-clonic seizures

Focal aware seizures

Focal impaired awareness seizures

Focal to bilateral tonic-clonic seizures

Absence seizures

Myoclonic seizures

Atonic seizures

Mixed seizure types
$\mathbf{N}=996$

$n=727(73.0 \%)$

$\mathrm{n}=50(5.0 \%)$

$\mathrm{n}=31(3.1 \%)$

$\mathrm{n}=89(8.9 \%)$

$\mathrm{n}=27(2.7 \%)$

$\mathrm{n}=20(2.0 \%)$

$n=10(1.0 \%)$

$n=42(4.2 \%)$

TABLE 2: Types of Seizures in All Patients

\section{TOTAL NUMBER OF PEDIATRIC PATIENTS}

Generalized onset tonic-clonic seizures

Focal aware seizures

Focal impaired awareness seizures

Focal to bilateral tonic-clonic seizures

Absence seizures

Myoclonic seizures

Atonic seizures

Mixed seizure types

$$
\begin{aligned}
& N=419 \\
& n=316(75.4 \%) \\
& n=12(2.9 \%) \\
& n=5(1.2 \%) \\
& n=22(5.2 \%) \\
& n=20(4.8 \%) \\
& n=12(2.9 \%) \\
& n=8(1.9 \%) \\
& n=24(5.7 \%)
\end{aligned}
$$

TABLE 3: Types of Seizures in Pediatric Patients

\begin{tabular}{|l|l}
\hline TOTAL NUMBER OF ADULT PATIENTS & $\mathrm{N}=577$ \\
\hline Generalized onset tonic-clonic seizures & $\mathrm{n}=411(71.2 \%)$ \\
\hline Focal aware seizures & $\mathrm{n}=38(6.6 \%)$ \\
Focal impaired awareness seizures & $\mathrm{n}=26(4.5 \%)$ \\
Focal to bilateral tonic-clonic seizures & $\mathrm{n}=67(11.6 \%)$ \\
Absence seizures & $\mathrm{n}=7(1.2 \%)$ \\
Myoclonic seizures & $\mathrm{n}=8(1.4 \%)$ \\
Atonic seizures & $\mathrm{n}=2(0.3 \%)$ \\
Mixed seizure types & $\mathrm{n}=18(3.1 \%)$
\end{tabular}

TABLE 4: Types of Seizures in Adult Patients

EEG was normal in 558 (56\%) patients while abnormal EEG was found in 438 (44\%) patients. Among abnormal EEGs interictal epileptiform abnormalities alone were found in $73.0 \%(\mathrm{n}=320)$, encephalopathy alone in $17.8 \%(n=78)$ and both interictal epileptiform abnormalities and encephalopathy in $9.2 \%(n=40)$ 


\begin{tabular}{|l|l|}
\hline TOTAL NUMBER OF PATIENTS & $\mathrm{N}=996$ \\
\hline NORMAL EEG & $\mathrm{N}=558(56.0 \%)$ \\
\hline ABNORMAL EEG & $\mathrm{N}=438(44.0 \%)$ \\
\hline Interictal epileptiform abnormalities & $\mathrm{n}=320(32.2 \%$ of total, $73.0 \%$ of abnormal EEGs) \\
\hline Encephalopathy pattern & $\mathrm{n}=78(7.8 \%$ of total, $17.8 \%$ of abnormal EEGs) \\
\hline Interictal epileptiform abnormalities and encephalopathy pattern & $\mathrm{n}=40(4.0 \%$ of total, $9.2 \%$ of abnormal EEGs) \\
\hline
\end{tabular}

TABLE 5: EEG Patterns

\section{Discussion}

The most common type of seizures overall in our study population was generalized onset tonic-clonic seizures which was found in $73 \%$ of study population, followed by focal to bilateral tonic-clonic seizures and focal aware seizures. The same three types of seizures dominated the presentation in adult population. The pediatric population presented most often with generalized onset tonic-clonic seizures, followed by mixed seizure types and focal to bilateral tonic-clonic seizures. These findings are similar to many other studies conducted in Pakistan and other countries. Aziz et al. conducted a study according to which the most common category of seizures was generalized onset tonic-clonic seizures followed by focal to bilateral tonicclonic seizures. The least common category of seizures was absence seizures (1\%). The cause of seizures could only be evaluated in $38.4 \%$ of their study population. Adequate management of epilepsy was only received by $27 \%$ of urban patients and $2 \%$ of rural patients [3]. The mean age of Aziz et al.'s study subjects was 13.3 years while the mean age in our study population was $21.4 \pm 3.2$ years. Aziz et al. conducted another epidemiological study in 1997 comparing the prevalence of epilepsy among population of Pakistan and Turkey. The most common type of seizures was similar among Pakistani and Turkish population [6].

According to previous studies the main cause of seizures in elderly is stroke which accounts for 30-70\% of cases. Cerebral tumors cause $10-15 \%$ of seizures in this population while metabolic disturbances are responsible for less than $10 \%$ seizures. Other causes include head injury, CNS infections and neurodegenerative disorders [7]. In children, the most common causes of seizures include high-grade fever, cerebral palsy, hypoxic-ischemic encephalopathy, infections, intracranial hemorrhage and hydrocephalus [8]. In our study, $58 \%$ of seizures were primary while $42 \%$ were secondary. This distribution was more even in children with $49.6 \%$ primary seizures and $50.4 \%$ secondary seizures; the gap widened in adults with $64.3 \%$ primary seizures and $35.7 \%$ secondary seizures. Among secondary causes, most common overall was neonatal encephalopathy followed by head trauma and CNS infections. Other causes found were cerebral tumors, stroke, metabolic encephalopathy, febrile seizures and cerebral malformations. Again the difference in children and adults was noticeable in causes of secondary seizures. Neonatal encephalopathy, CNS infections and febrile seizures represented the most frequently presenting causes of secondary seizures in children. In adults the top three causes of secondary seizures were head trauma, cerebral tumors and stroke.

Kaur et al. conducted a study on the causes of seizures among adults admitted to a tertiary care setup in India. The most common cause of seizures in their study population was stroke followed by idiopathic causes and CNS infections. Cerebral tumors accounted for $10 \%$ of cases while metabolic disturbances were responsible in $12 \%$ patients. The most common cause of generalized onset tonic-clonic seizures was idiopathic while the most common cause of focal seizures was stroke. Status epilepticus was most often caused by metabolic derangements. They concluded that advanced age led to reductions in incidence of generalized seizures and focal seizures became more predominant as age progressed [9]. Mac et al. conducted a systemic review on the causes of epilepsy in Asia. Prevalence data from 11 different countries was analyzed. According to this study, head trauma, stroke, CNS infections and trauma during birth account for the most common causes. They concluded that diagnostic and therapeutic modalities were limited in many regions [10].

In our study, the mean age was $21.4 \pm 3.2$ years. In studies conducted in Pakistan and other underdeveloped countries in Asia, the mean age for epileptic patients lies between 15-20 years. However in developed countries epilepsy has a bimodal distribution. The first peak is after few years of birth whereas the other peak occurs after 65 years of age [11]. Most of our patients were male. Khan et al. conducted a study on the prevalence of epilepsy in children in 2019 in Karachi. He concluded that the prevalence was greater in males compared to females. Epilepsy was present in $22 \%$ of his study population with generalized onset tonicclonic seizure as the most common type [12]. 
Ullah et al. in 2018 conducted an epidemiologic study on the characteristics of epileptic patients in Khyber Pakhtunkhwa (KPK) province of Pakistan. The mean age of their study population was $18 \pm 8$ years with majority of patients being male. Most patients from this study belonged to low socio-economic status. The characteristics associated with poor response to management included low socio-economic status, noncompliance to therapy and generalized onset tonic-clonic seizures [4].

Our study found that patients with epilepsy represented $10.5 \%$ of the total patient turnover in neurology department of our hospital. Considering the vast number of neurological illnesses this number looks a bit high. This can probably be explained by the fact that internal medicine departments and general practitioners often feel confident in treating some commonly presenting neurological diseases like headache and stroke, but most of the epilepsy patients are referred to neurology services. We also looked into the EEG patterns of our patients and found that $56 \%$ of them had normal EEG. $32.2 \%$ patients had interictal epileptiform abnormalities, $7.8 \%$ had encephalopathy pattern and $4 \%$ had both interictal epileptiform abnormalities and encephalopathy pattern. McGinty et al. found that $54.4 \%$ of their patients had normal EEG, 24.6\% had non-specific abnormalities and 21.1\% patients demonstrated interictal epileptiform abnormalities [13].

The limitation of this study is that it represents the patients presenting to a single hospital and therefore generalization over the whole population may not be appropriate. Major strength of this study was a significant sample size spread over two years that minimize the chances of false representation of the actual state of affairs.

Neonatal encephalopathy and head trauma were responsible for secondary epilepsy in majority of pediatric and adult population respectively in our study. Maternal and neonatal health has always been a major concern in Pakistan. This study, by finding neonatal encephalopathy as the leading cause of secondary epilepsy in children, reinforces the need for improving this crucial aspect of society health. Bike riders are frequently seen without helmets on all the major roads of Karachi. Head trauma was consequential for the most number of adult patients in this study; this underpins the need for creating awareness and perhaps implementing more stringent measures to eliminate this harmful habit among the population. Further studies on the causes and types of epilepsy in Pakistan are required as these factors differ between populations and hence the management strategies also differ consequently.

\section{Conclusions}

This study provides an insight into an under-researched area of epilepsy in Pakistan. The causes and types of seizures dictate the best possible treatment options. We found that the most common cause of seizures in our overall study population was primary seizures, though primary and secondary seizures were more evenly present in children. Among secondary causes neonatal encephalopathy stood out as the most common cause in children; head trauma was the predominant cause in adults. Most common type of seizures overall and in adults was generalized onset tonic-clonic seizures, followed by focal to bilateral tonic-clonic and focal aware seizure types. Pediatric patients presented most often with generalized onset tonic-clonic seizures. Mixed seizure types and focal to bilateral tonic-clonic seizures were the next most common type of seizures in children. Further studies are needed in Pakistan so that conclusive data is accumulated in this regard to aid in making better decisions to combat this common disease.

\section{Additional Information \\ Disclosures}

Human subjects: Consent was obtained by all participants in this study. Animal subjects: All authors have confirmed that this study did not involve animal subjects or tissue. Conflicts of interest: In compliance with the ICMJE uniform disclosure form, all authors declare the following: Payment/services info: All authors have declared that no financial support was received from any organization for the submitted work. Financial relationships: All authors have declared that they have no financial relationships at present or within the previous three years with any organizations that might have an interest in the submitted work. Other relationships: All authors have declared that there are no other relationships or activities that could appear to have influenced the submitted work.

\section{References}

1. Kasper D, Fauci A, Hauser S, Longo D, Jameson J, Loscalzo J: Harrison's Principles of Internal Medicine, 19e . Shanahan JF, Davis KJ (ed): McGraw-Hill Education, New York; 2015.

2. Thijs RD, Surges R, O'Brien TJ, Sander JW: Epilepsy in adults. The Lancet. 2019, 393:689-701. 10.1016/S0140-6736(18)32596-0

3. Aziz H, Ali SM, Frances P, Khan MI, Hasan KZ: Epilepsy in Pakistan: a population-based epidemiologic study. Epilepsia. 1994, 35:950-958. 10.1111/j.1528-1157.1994.tb02539.x

4. Ullah S, Ali N, Khan A, Ali S, Nazish HR: The epidemiological characteristics of epilepsy in the province of Khyber Pakhtunkhwa, Pakistan. Front Neurol. 2018, 9:845. 10.3389/fneur.2018.00845

5. Scheffer IE, Berkovic S, Capovilla G, et al.: ILAE classification of the epilepsies: position paper of the ILAE Commission for Classification and Terminology. Epilepsia. 2017, 58:512-521. 10.1111/epi.13709 


\section{Cureus}

6. Aziz H, Güvener A, Akhtar SW, Hasan KZ: Comparative epidemiology of epilepsy in Pakistan and Turkey: population-based studies using identical protocols. Epilepsia. 1997, 38:716-722. 10.1111/j.1528-

1157.1997.tb01242.x

7. Li SH: Epidemiology and etiology of seizures and epilepsy in the elderly in Asia . Neurol Asia. 2004, 9:31-32.

8. Pavone P, Corsello G, Ruggieri M, Marino S, Marino S, Falsaperla R: Benign and severe early-life seizures: a round in the first year of life. Italian J Pediatr. 2018, 44:54. 10.1186/s13052-018-0491-z

9. Kaur S, Garg R, Aggarwal S, Chawla SP, Pal R: Adult onset seizures: clinical, etiological, and radiological profile. J Family Med Primary Care. 2018, 7:191-197. 10.4103/ifmpc.jfmpc 32216

10. Mac TL, Tran DS, Quet F, Odermatt P, Preux PM, Tan CT: Epidemiology, aetiology, and clinical management of epilepsy in Asia: a systematic review. The Lancet Neurol. 2007, 6:533-543.

11. Beghi E, Giussani G: Aging and the epidemiology of epilepsy. Neuroepidemiology. 2018, 51:216-223. $10.1159 / 000493484$

12. Khan J, Ghoto MA, Dayo A, Arain MI, Parveen R, Khan K, Memon AA: Prevalence and clinical evaluation of epileptic disorders in children at a tertiary care centre in Karachi, Pakistan. Lat Am J Pharm. 2019, 38:10201023.

13. McGinty RN, Costello DJ, Kinirons P, McNamara B: Diagnostic yield of routine EEG in adults with active epilepsy. Ir Med J. 2019, 112:851. 